\title{
Jiao Zhou Yangko during the New Rural Construction
}

\author{
Zhao Lei \\ Qingdao Huanghai University \\ Qingdao, Shandong, 266427
}

\author{
Zhang Xipeng \\ Qingdao Huanghai University \\ Qingdao, Shandong, 266427
}

\begin{abstract}
The new rural construction refers to the construction of the economic, political, cultural and social aspects of rural areas under the socialist system, in accordance with the requirements of the new era, and finally realizes the rural construction into economic prosperity, perfect facilities, beautiful environment and civilization the goal of the new socialist countryside. Jiao Zhou Yangko as the most extensive basis of the rural mass sports project, in the construction of a socialist harmonious society and new rural construction, to find an opportunity for their own development, and this paper is in this context through the overview of Jiao Zhou Yangko, Giving its new features, and put forward the corresponding countermeasures. Enrich the spiritual and cultural life of the masses in the construction of new countryside, and provide practical guiding significance for the better sustainable development of Jiao Zhou Yangko.
\end{abstract}

Keywords-New Rural Construction ; Healthy China ; Jiao Zhou Yangko

\section{THE DESCRIPTION OF JIAOZHOU YANGKO}

\section{A. Background of the New Times of Jiaozhou Yangko}

Folk art is a significant feature of Jiaozhou Yangko, and it is the portrayal of the real artistic life. Jiaozhou Yangko begins in rural areas in the Xianfeng Period, Qing dynasty. Until now it still maintains the original ecology and deep rural flavor. As the first Yangko of the three Yangkos in Shandong, Jiaozhou Yangko has accompanied Chinese people in a long history, more than one century. Up to now it has had more fashion factors and wide bodybuilding significance. Under the background of the new rural construction and healthy China concept, Jiaozhou Yangko holds a position of great social responsibility, which includes assisting healthy China. On the contrary, this background of the new times has provided rich vegetative soil for Jiaozhou Yangko.

\section{B. Historical Changes of Jiaozhou Yangko}

Based on farming culture, Jiaozhou Yangko is grown in the rich cultural environment of Qi. As a traditional folk art, it is an absolute peasant art with outstanding features, such as the spontaneous participation, free emotional catharsis, conscious joining, entertaining themselves and voluntary changing. From a social historic point of view, Jiaozhou Yangko originates from the end of feudal society, which is during the war which broke out at the end of Ming dynasty and the beginning of Qing dynasty. It has changed during the collapse of feudal

Jiaodong Yangko and Communications among the villages. No. J16WI03. society and the starting of democratic revolution. It has thrived before the break out of the Anti-Japanese War and declined in the eight years of war and three-year civil war; after the founding of the people's republic of China, it has experienced the flourishing of the early years of the new nation, the decay of "Cultural Revolution", the expecting of "Reform and Opening-up" and the protection and discovery of successful application for world heritage.

\section{Peasant Art Characteristic of Jiaozhou Yangko}

1) Scientificity and Fitness Nature of Jiaozhou Yangko

As a folk dancing art, Jiaozhou Yangko has stretching and decent movements. It can cultivate peasants' taste and strengthen their physical fitness.

Jiaozhou Yangko is not only a form of folk art, but also a traditional ethnic sport. "The function of traditional ethnic sports is determined by the essence of sports, which is able to meet the needs of human amusement and promote the sound improvement of human mind and body. And this is the essential function of sports." One of the essential functions of sports is fitness function. Therefore, as a traditional ethnic sport, Jiaozhou Yangko is beneficial to physical and psychological health, and it owns the fitness function.

Firstly, Jiaozhou Yangko owns the function of strengthening the body and promoting physical well-being. To the accompaniment of music, Jiaozhou Yangko is a kind of sports with lower strength and higher quantity. It can improve blood circulation and promote the respiratory, as well as exercise the muscles. A lot of information show that Yangko can decrease the TC, TG and LDL-C in the blood of the middle-aged and aged people, and decrease the fat deposition in the vessel wall. Besides, it can improve the cardio vascular function and increase the HDL-C of the middle-aged and aged people, through which the incidence rate of cardiovascular system has been effectively reduced. Jiaozhou Yangko, as a kind of sports fitness mean with high quality and low load, it is a body-building item suitable for people of all ages, and it has positive effect in enhancing people' s comprehensive quality.

\section{2) Entertainment and Aesthetic Character of Jiaozhou} Yangko

As a dance form, Jiaozhou Yangko is a form of folk art. Its charming lies in the characteristic of self-entertainment and entertaining others, as well as a variety of aesthetic feeling; Yangko can enable performers to fully release their selfemotion and upgrade their soul. With the fast or slow, strong or 
weak and fluent instrumental rhythms, the emotion of "no matter how secret of your inner activities, they will be reflected from your behaviors, expressions or eye expressions". At the meanwhile, the viewers will be affected by the performers. Their inner world will be influenced and thus a pleasant feeling will be appeared. Yangko performance will create a harmonious beauty of self-appreciation and appreciation for performers and viewers. Its figurativeness, infectivity, originality and craft are the source of aesthetic appreciation. The double entertaining functions of self-entertainment and entertaining others are achieved.

\section{THE FUNCTION OF JIAOZHOU YANGKO}

\section{A. Jiaozhou Yangko has Realized the Political Interaction and Cultural Exchange}

Social Development uses economy as the linkage, backed with culture. Culture has increasingly become an essential index for the reflection of comprehensive competitiveness of a nation or an area. Enhance the soft power of culture has already become important content to enhance the core competence of an area. As one of the major characteristic culture, Jiaozhou Yangko has developed its Mao Opera brand, and wished to make it bigger and stronger. The cultural products and cultural service of Jiaozhou Yangko has occupied the market, which has been well received by the mass. In the political and cultural development process, Jiaozhou Yangko has played important roles in the following aspects: making contributions to the allround well-off society, uniting and bringing political thought height to the full implementation of work arrangement and gathering strength to speed up every items of cultural construction.

\section{B. Jiaozhou Yangko has Promote the Economic Industrialization Development}

The economic value of Jiaozhou Yangko owns the characteristics of anaclisis, potentiality and peculiarity. The economic value can be developed only by attaching to some definite mechanism and vector, which can either be the art and craft or the art form. The value of Jiaozhou Yangko cannot play its role without the vectors; the development of economic value or Jiaozhou Yangko requires some conditions. It they do not have such conditions or the conditions are not enough, and its economic value is only a possibility.

The reasons why Jiaozhou Yangko could promote the economic industrialization development lie on its unique national characteristic. At the meanwhile, government has provided advantaged objective conditions for the development of Jiaozhou Yangko. Therefore, the economic industrialization development of Jiaozhou Yangko has been promoted. For example, Yangko festival, which is hold once every two years and starts in May. It is last for three days. The affiliated food court lasts for one week. It has attracted people come to visit and travel.

\section{Jiaozhou Yangko has Speed up the Development of National Fitness Project}

Nationwide fitness campaign should be widely carried out. We should promote the physical activities of emphatic crowds and promote the deep integration between national fitness and public health. And one district, one brand, one characteristic should be reflected. Jiaozhou Yangko has a large population base, and owns some fitness service personnel. They have regular contest performance and fixed stadium. During the Olympic Games, there are six Jiaozhou Yangko teams; nearly 600 people took part in the "Do the Yangko Dance and Welcome the Olympic Games" in the May Fourth Square of Qingdao. It is spectacular by seeing the number of participants. Nowadays, Jiaozhou Yangko has entered into schools, enterprises, institutions and communities. They have conducted the Yangko tour in every city area, villages and cultural squares. The sense of honor of the entire people and cultural confidence has been enhanced, and the Qingdao national fitness project has been accelerated. The fresh blood has been infused into the sustainable development of national fitness project.

\section{DeVEloping PREDiCAMENT FACED By JiAOZHOU YANGKO IN THE PROCESS OF NEW RURAL CONSTRUCTION}

Secondly, Jiaozhou Yangko owns the function of promote people's psychological health. Physical training is a good recipe for psychology treatment. However, as a traditional sport, Jiaozhou Yangko is a way for expressing and adjusting, which is able to relieve the working pressure of youth and the aged people and help aged people to resolve their inner loneliness, as well as prevent and cure some mental disease. To the accompaniment of music, people may do the Yangko dance and relax their mind, as well as cultivate their taste and feel alive and at their best.

The successful application for World Heritage of Jiaozhou Yangko and the substantial support and protect by local government have provided some specific institutional guarantee for its benign development. However, under the background of current economic reform and national fitness project, there are some problems showed in the development of Jiaozhou Yangko.

\section{A. Venue and Equipment Need Urgent Perfection}

Economic development determines superstructure and restricts the spiritual and cultural life. The general economic level of Jiaozhou ranks the forefront in the districts and cities of Qingdao. However, economic backward occurred in some communities and villages. There are no venues, equipment nor cultural square. More seriously, there is no equipment in some villages. If Jiaozhou Yangko wants to develop consumedly, some hardware conditions should be used as the basis and some software conditions should be used as support; the population of Yangko dancers cannot be enlarged if the venues and equipment are not perfected. It will even meet or is just suffering the bottleneck period of the development of Yangko population. 
B. The Operation Difficulties of Civic Yangko Theatres Led by Insufficient or Delayed Qualified Teachers and Fund

There are many Jiaozhou Yangko teams. However, they are still in the early stage with some difficulties. Backstage crew are exceeding deficient. Usually there are no specific guide teachers. They cannot find teachers before competition or they cannot find good teachers. Some Jiaozhou Yangko teams use one music band, which has restricted the possibility of the small villages taking part in the competitions at the same time. Most of the other Jiaozhou Yangko teams do not have backstage crew. The performance only depends on the voice recording equipment, and the original ecology characteristic of Yangko has been reduced. There is shortage in rehearsal schedule. Most of the Yangko teams do not have rehearsal, nor training. They only have massed learning and rehearsal before large-scale performance or competitions. The enjoyability of Yangko will be certainly influenced; for example, there are four male Yangko dancers in the Second Village of Nanzhuang, and three of them are working in enterprises. They are inconvenient for asking for leaving. Village committee asks for leave for them, and 50 to 100yuan will be deducted based on the length of the leave. Government does not provide the fund support, and the Yangko dancers who are working in enterprises have to maintain their lives. Thus the mass learning, rehearsal time and frequency have been largely reduced and the theatres cannot be run normally.

\section{DeVeloping StRategies of JiaOzhou YangKo in THE PROCESS OF THE NEW RURAL CONSTRUCTION}

\section{A. Constructing Cultural Square and Increase the Operational Rate of Venue and Equipment}

Based on the geographic location of local community and village, each community should construct the independent or joint cultural square with considering their own economic level. Yangko training venue and performance venue should be arranged properly and the perfection of related Yangko equipment should be accelerated.

\section{B. Making Use of the Universities and Colleges and Cultivating New Professional Yangko Teachers}

Professional settings and training should be arranged for some Jiaozhou Yangko specialists and guide teachers. We should promote the development of Jiaozhou Yangko in communities and increase the mass base. We should combine the artistic characteristics of rhythmic gymnastics and aerobics to enrich the connotation of Yangko. The aesthetic experience of Yangko performance will be enhanced, through which the audience will receive visual shock directly.

\section{Strengthening the Commercial Performance among Areas, Making up for the Shortage of Fund and Exploring the New Mode of Developing Together}

It is essential to establish the Yangko competition or performance committee, strengthen the Yangko cultural exchange among each area and develop together. Bonus system should be established before competitions or performance. Government should provide the opportunities for performing Yangko, such as the company ceremony and public welfare performance, through which the communication exchanges of competition and performance have been increased and the shortage of fund has been compensated. Besides, Yangko culture has been popularized.

\section{CONCLUSION}

Jiaozhou Yangko stays in the time of national fitness and in the progress of the new rural construction. It has played its due role to enrich the spiritual and cultural life of the people who lived in this city, this province or even the whole country and promote the harmonious of the whole village. Functional government departments should pay more attention to the policy implementation and make sure of the sustainable development of Jiaozhou Yangko. Through promoting and popularizing Jiaozhou Yangko, the positive and important role will be played in the socialism new countryside construction. The excellent national culture with Chinese characteristics will be displayed to the world and the pace of healthy China has been promoted.

\section{REFERENCES}

[1] Wang Zhaoqiu. Jiaozhou Yangko Textbook [M]. Beijing: China Federation of Literary and Art Circles Press, 2007, 3.

[2] Pan Zhitao. Chinese Folk Dance Teaching Material and Teaching Methods [M]. Shanghai: Shanghai Music Publishing House, 2001, 89.

[3] Research Group. Investigation and Research of Sports Current Situation of Chinese Mass [M]. Beijing: Beijing Sport University Press, 1998.

[4] Sun Yongping. The Study of Sociology of Shenyang Yangko Activities Sports [J]. Journal of Shenyang Physical Education Institute, 2006, (2): 15

[5] Wang Zhaoqiu. Jiaozhou Yangko Textbook [M]. Beijing: China Federation of Literary and Art Circles Press, 2007, 3. 